\title{
Féeries
}

Études sur le conte merveilleux, XVII ${ }^{\mathrm{e}} \mathrm{XIX} \mathrm{X}^{\mathrm{e}}$ siècle

\section{« Rédifier » les contes de Grimm : Chants populaires de Philippe Beck}

Rebuilding/Re-Edifying Grimm's Tales: Philippe Beck's Popular Songs

Jean-François Perrin

\section{(2) OpenEdition}

1 Journals

Édition électronique

URL : http://journals.openedition.org/feeries/766

DOI : $10.4000 /$ feeries.766

ISSN : 1957-7753

Éditeur

UGA Éditions/Université Grenoble Alpes

\section{Édition imprimée}

Date de publication : 31 octobre 2010

Pagination : 161-178

ISBN : 978-2-84310-182-3

ISSN : 1766-2842

\section{Référence électronique}

Jean-François Perrin, « «Rédifier » les contes de Grimm : Chants populaires de Philippe Beck », Féeries [En ligne], 7 | 2010, mis en ligne le 31 juillet 2011, consulté le 08 septembre 2020. URL : http:// journals.openedition.org/feeries/766 ; DOI : https://doi.org/10.4000/feeries.766

\section{(c) Féeries}




\title{
"RÉDIFIER" LES CONTES DE GRIMM : CHANTS POPULAIRES DE PHILIPPE BECK
}

\begin{abstract}
T L S'AGIRA ICI DE LA PRÉSENTATION d'une entreprise poétique contemporaine en langue française, consistant en la récriture versifiée de 72 contes des frères Grimm par un poète considéré comme l'un des chefs de file de sa génération. Philippe Beck est né en 1963. Il enseigne la philosophie et développe depuis 1996 une production déjà considérable puisqu'il avait publié une douzaine de livres avant celui dont je vais m'occuper : Chants populaires, paru en 2007 aux Éditions Flammarion. Avec un ouvrage antérieur: Poésies didactiques (200I), ce livre inscrit une tentative audacieuse et entêtée pour articuler aujourd'hui une réaffirmation du lien entre pensée et poésie, sous horizon de quête de vérité : conception certes méditée à partir du romantisme allemand, mais attentive à l'exigence de forme qu'implique artistiquement - envers et contre tout - le chaos de notre époque. Ainsi, la récriture des contes selon Beck prend en compte la teneur mythique éparpillée dont on les crédite depuis les Grimm, en même temps que la nécessité de mesurer la distance qui nous en sépare. On résumera cela en deux brèves citations :
\end{abstract}

Si mort est le premier mythe,

alors il a l'air

du Discours du tout près.

Cerbère garde le pays

Sans Retour

(juste à côté),

et il a des petits historiés.

(Poésies didactiques, p. I3.)

Ma mère l'Oie est plombée.

L'écrivain découpe à livre ouvert

et il ne copie pas la culture

fidélisé,

il travaille comme elle - 
elle est trouvée

elle se trouve.

(Ibid., p. 23.)

Le titre Chants populaires est une citation malicieuse de la préface des frères Grimm à la première édition (I8I2) de leur fameux recueil de contes :

(Après une tempête catastrophique, il arrive qu'en) un petit coin de champ [...] quelques épis isolés sont encore debout [...]. Quand ils seront mûrs et pleins, des mains pauvres et pieuses viendront les chercher [...] les porteront chez elles [...] où ils seront peut-être la seule semence pour l'avenir. C'est le sentiment que nous avons quand nous considérons la richesse de la poésie allemande, et que nous voyons ensuite que, de tant de choses, rien ne s'est conservé vivant, que même le souvenir en a été perdu et qu'il ne reste plus que des chants populaires et ces innocents contes du foyer. Les lieux près du poêle, du fourneau de la cuisine, les escaliers de grenier, les jours de fête que l'on célèbre encore, les chemins [...] et les forêts, dans leur silence, et, avant tout, l'imagination inaltérée, sont les haies qui les ont préservés et qui les ont transmis d'une époque à l'autrer.

Dans ce paragraphe d'allure très «littéraire» sont condensées une série de thèses propres aux frères Grimm et plus largement au romantisme du cercle d'Iéna à la fin du XVIII ${ }^{\mathrm{e}}$ siècle. La préface développe ainsi l'idée de liens supposés directs de ces contes et chansons populaires avec une tradition épique allemande perdue, un mythe originel fondateur de la Germanité; puis celle d'une source éternelle poétiquement irrésistible, naturellement connectée à l'imaginaire et à la sensibilité humains et foncièrement didactique. À quoi se lie l'euphémisation sous couvert d'innocence primitive, de la profonde relation des contes au mystère du Mal. Enfin, elle affirme que le recueil publié est retranscrit de la pure tradition orale paysanne allemande : «À quelques exceptions près [...] tout a été collecté presque exclusivement en Hesse et dans les régions du Main et de la Kinzig, dans le comté de Hanau d'où nous sommes originaires, d'après la tradition orale.» Or on sait aujourd'hui que les informatrices des frères Grimm étaient plutôt des femmes cultivées, familières de la culture française, et qu'ils ont agi en écrivains, sélectionnant, recomposant, hybridant en vue d'une idée du conte populaire allemand, d'un style de l'authenticité primitive, d'une sorte d'épure fictionnelle de ce que devait être un tel conte.

C'est ce que rappelle Philippe Beck dans l'Avertissement inaugural de son livre, à propos de la copie première des contes, retrouvée en 1920 :

I. Contes pour les enfants et la maison collectés par les frères Grimm, trad. et éd. de N. RimassonFertin, Paris, José Corti, 20Io, t. I, p. 472-473. 
Elle atteste une stylisation et une mythification des sources orales notamment paysannes. [...] Près de la moitié des contes [...] peuvent avoir une autre source que la tradition orale des paysans et artisans allemands. Une source écrite. [...] Les contes relèvent de la "poésie d'art» et non de la seule "poésie de la nature». [...] Quant à la "stylisation" ou «adaptation" (nous dirions version ou récriture), Jacob Grimm dit encore: «Tu ne peux pas faire de récit parfaitement conforme, de même que tu ne peux casser un ouf sans qu'un peu de blanc reste collé à la coquille [...]. Pour moi, la vraie fidélité dans cette image, ce serait de ne pas casser le jaune de l'œuf.» (p. 8)

Le travail de Beck inscrit d'emblée une récriture, aujourd'hui, de la récriture romantique, non pas pour la dénoncer stricto sensu mais pour en retrouver l'axe fondamental, et non comme imitation mais comme transposition d'une recherche - ainsi située sur la $4^{\mathrm{e}}$ de couverture de ses Poésies didactiques (200I) :

Dans le livre sur la poésie naïve et sentimentale, Schiller a cette phrase : «on attend encore un poème didactique où la pensée elle-même serait et demeurerait poétique.» C'est comme une réponse à la future phrase ambiguë de l'Esthétique de Hegel : «à strictement parler, la poésie didactique n'est pas de la poésie.»

Cette conception de la pensée en tant qu'inévitablement poétique lorsqu'elle "pense vraiment (en vérité) ${ }^{2}$ » est effectivement au cœur du projet romantique jusqu’à Hölderlin; elle nourrit aujourd'hui le projet de Beck. En voici l'une des formulations : "On appelle pensée l'espace ou de la prose trouve une exactitude baptisée poème ${ }^{3}$.» En voici une autre qui dit le rapport musique/sens dans cette expérience, autant pour le lecteur que pour l'auteur : "Ce qu'il y a à dire se pratique, c'est déjà une suite rythmique. Le poème est toujours didactique dans le rythme, qui est rythme de l'idée " (ibid., p. 47), "car la vérité que le poème dit est non seulement une vérité en rythme, mais une vérité essentiellement rythmique ${ }^{4}$.

Il n'est pas question ici de développer la problématique générale qui sous-tend une telle posture (où Beck travaille en dialogue avec des courants bien précis de la pensée et de la poésie contemporaine); outre le livre d'entretiens cités, on renverra notamment à l'ouvrage bien connu de Philippe Lacoue-Labarthe et Jean-Luc Nancy : L'Absolu littéraire. Théorie de la littérature du romantisme allemand (Seuil, 1978). L'essentiel, dans le

2. Ph. Lacoue-Labarthe, "Entretien sur Hölderlin avec Patrick Hutchinson», Europe, n $^{\circ} 973$, mai 20IO, p. 49.

3. Beck, l'impersonnage, rencontre avec Gérard Tessier, Paris, Argol, 2006, p. IIo. (Désormais B. I.)

4. Entretien avec D. Christoffel, disponible sur <http://www.doublechange.com/issuez/beckintfr.htm> [consulté le 8 septembre 20Io].

5. Voir aussi deux dossiers : "Maintenant Philippe Beck», Le Polygraphe, $\mathrm{n}^{\circ}{ }_{13}$-I4, été 2000 , p. 20II-2045; «Philippe Beck : une poésie recommence», Il particolare, $\mathrm{n}^{\text {os }} 7$ et 8, 2002. 
cadre limité de cette étude, est d'avoir rappelé un dispositif qui place le travail poétique du côté de cette fameuse "poésie objective» réclamée par Rimbaud, et dont Philippe Beck postule qu'elle est «le nom d'un récit général égal au raisonnement sensible dont chacun est capable» (B. I., p. 79). Pour autant, les Chants populaires travaillent sur des objets (les contes de Grimm) «qui ne sont pas des fables» en ce qu'ils maintiennent latent un travail du négatif, au lieu que les fables (selon Hegel) présentent des "énigmes toujours accompagnées de leurs solutions" ${ }^{6}$. Ainsi, il convient de "dé-moraliser" ces contes en détournant ou critiquant "la matière passée, bronzée, / débrimée, / car beaucoup est à dire / sur la façon de tout dire / de certains anciens, / ou d'anciens modernes ${ }^{7}$ ». Ce que résume cette formule d'Inciseiv: "J'appelle philosophie / l'art d'être dans la poésie / et d'avoir en poésie / beaucoup d'impersonnalité ${ }^{8}$." Les Chants populaires se situent dans cette perspective : chants à penser et récitatifs $\operatorname{secs}^{9}$, ce sont des chants objectifs qui opposent la poésie de pierre (antilyrique) à l'humidité lyrique propre à la "rhumanité». (Beck a forgé ce néologisme à partir de «surhumanité» pour formuler ce qui doit précisément échapper à l'inhumain de l'héroïsme) :

Poème est enseignement ou rappel de la condition humaine. Condition qui se déshumanise dans l'Aveuglant, la lumière du jour social. Or il y a dans la lumière de la dépendance une chance de rhumanité. Le héros c'est la condition inhumaine, c'està-dire la condition qui s'oublie, s'aliène, s'oblige à l'indéfinition et à la redéfinition. (B. I., p. 79.)

Le travail des Chants populaires sera donc «de façonner la leçon future qui s'appuie sur de beaux enseignements épuisés», telle "une fable d'Ésope désormais inutilisable» à une époque où «le cours de l'expérience a chuté» (Walter Benjamin) ${ }^{\text {Io }}$. Quant à l'enracinement mythique de l'affaire, il faut certes rappeler le mythe romantique de la collecte/stylisation selon Wilhelm Grimm : "Les éléments mythiques sont des débris qui ressemblent aux petits morceaux d'une pierre précieuse éclatée, qui seraient éparpillés sur le sol recouvert d'herbe et de fleurs et que seul un regard plus perçant que les autres peut découvrir» (Avertissement des Ch. p.) — mais

6. Ph. Beck, Chants populaires, Avertissement, Paris, Flammarion, 2007, p. 9. (Désormais Ch. p.)

7. Ph. Beck, Poésies didactiques, Théâtre typographique, Courbevoie, 200I, p. 24 et 38. (Désormais P. did.)

8. Ph. Beck, Inciseiv, Nantes, MeMo, 2000, p. 54.

9. Ph. Beck, Le Fermé de l'époque, Marseille, Al Dante, 2000, p. 32.

IO. Ph. Beck, entretien avec E. Laugier, Le Matricule des anges, nº 8I, 2007, p. 44. (Désormais MdA.) 
sans jamais négliger d' "imaginer qu'il s'agit d'une phrase écrite il y a deux siècles environ ${ }^{\mathrm{II}} »$. Autrement dit, comprendre, pour notre temps «l'irréductible immoralité des catastrophes, que la culture, fondée sur la barbarie, a sinon préparées, du moins cautionnées en partie et interdit de penser». Ainsi le dit en vers le Finale des Chants populaires:

Langue se délie en barbarie.

Sur le chemin des épingles

ou le chemin des aiguilles.

Dans un faux jour aussi.

Depuis les grandes bassesses. (p. 217)

Or le merveilleux est souvent dur, remarque Beck :

Si un conte fait peur, c'est pour que les gens, et les enfants d'abord, fassent attention aux dangers. Mais si la tradition a permis les dangers qu'elle proposait d'éviter, alors il ne faut pas recopier les contes; il faut en montrer les nervures, les ressorts, les beautés séduisantes. Le conte est une sirène. Chacun est Ulysse. ( $M d A$, p. 44.)

Ainsi les Chants populaires assèchent les contes, les essorent, les refont :

Le chant $[\ldots]$ serre, trie et refond en lui des données du conte, en efface, invente de nouveaux personnages [...] ou renomme les personnages. [...] Le chant intense est un chant qui [...] détend et retend les données, fait l'unité des données anciennes ou antiques et des données de la vie moderne. ( $M d A$, p. 45.)

Dans l'analyse qui va suivre, j'ai choisi de travailler (ou d'interpréter) un seul poème de ce recueil. Je l'ai choisi parce que tout le monde a en mémoire le conte qu'il récrit (Blanche-Neige), ce qui rend plus sensible, je l'espère, les variations et ruptures qu'introduisent la langue et le vers de Beck; et aussi parce que son titre «Réversibilité» me parait emblématique de toute l'entreprise, dans la mesure où il joue de l'équivalence postulée par la préface de I8I2 aux Kinder- und Hausmärchen, entre «chants populaires» et "contes du foyer». Ce Chant, numéroté 2I dans le recueil, est donné à la suite; quant au texte de Blanche-Neige, on le trouvera en annexe (Beck paraît avoir travaillé sur la traduction d'Armel Guerne) ${ }^{12}$, muni d'un certain nombre de repères destinés à faciliter la lecture du travail de condensation/réinvention drastique dont il fait l'objet.

2I. Réversibilité

En hiver des flocons descendent

I

comme des plumes

II. Ibid.

I2. On verra que le suivi de la récriture est très littéral par rapport à la traduction de Guerne, ce qui n'exclut pas, Beck étant germaniste, une autre strate de travail directement sur l'allemand. 
d'oiseau discret.

Femme à la fenêtre noire

donne trois gouttes de sang

à Neige.

C'est un coquelicot de soi,

aux pétales séparés.

Elle a bientôt une enfant à trois couleurs.

Une couleur lui donne son nom.

Mère Suivante est peuplée.

Elle a un miroir qui dit si elle est singulière.

Miroir amagique.

L'enfant grandit. Elle est comme le jour.

L'interrogatoire du miroir

crée de nouvelles couleurs dans le cour

de la mère suivante :

jaune et vert.

Cœur tangue dans le ventre.

Mère successive.

Orgueil pousse en elle,

comme herbe sombre.

Au loin dans la forêt, enfant

comme le jour est laissée.

Pompe animale est humanisée.

Pompe de discours et désir.

Dedans remplacé.

Réaffecté.

Neige semble éliminée.

Mais dans la Forêt,

Neige retourne les feuilles. Nuit tombe.

Elle trouve une maison

Miniature. Comme Alice?

Nappe blanche et draps blancs dedans.

Est-ce le Hollandais Volant?

Un navire à bascule?

Nuit noire couvre montagnes

et ses mines futures.

Mère suivante s'habille.

Elle vend du bel et bon.

Lacet coloré, peigne rond,

pomme à deux couleurs.

Blanche et rouge.

Blanche-Neige est presque morte,

ou morte officiellement. Décolorée. Miroir

dit la vérité froidement.

Et l'antichambre réelle.

Elle n'est pas dans la terre noire.

Elle est intacte dans le verre. 
Des bêtes la pleurent.

Blanche a l'air de dormir infiniment.

Elle a un pré-sourire.

Elle est admirée d'un

qu'elle aime immédiatement;

ou dans une brève suite de moments

commence l'élan.

Et le cœur de la «mère»

est cuit;

envie a brûlé ses mouvements.

Vie dure.

60

D’après "Blanche-Neige».

\section{«Les Chants populaires dessèchent les contes»}

Le conte de Grimm subit ici une vigoureuse cure d'amaigrissement : quatre pleines pages de prose sont en effet ramassées en une colonne de vers qui occupe un tiers de page; mais dans cette colonne elle-même, $2 / 3$ des vers (v. I à 36) ramassent la matière narrative de la seule page I de la version en prose (de la conception de Blanche-Neige à son installation dans la maison des nains). Ainsi de vastes séquences de ce qui suit sont-elles résumées en quelques vers : par exemple, les trois tentatives de la marâtre (I page $1 / 2$ ) sont résumées en 6 vers (v. 37-43). Ce qui fait aussi fondre la matière verbale, c'est l'élision systématique de l'ensemble des dialogues (la moitié du texte dans la version en prose), qu'il s'agisse des entretiens de la reine avec son miroir, ou de l'ensemble des dialogues liés aux personnages masculins (chasseur, nains, amant) — lesquels sont d'ailleurs éliminés du poème à l'exception du dernier, cependant réduit à la portion plus que congrue (v. 53); qu'il s'agisse encore des pièges verbaux tendus à Blanche-Neige par la marâtre déguisée. Ce qui demeure de la matière originale consiste pour l'essentiel en prélèvements lexicaux et en paraphrases.

On saisit la procédure de récriture dès l'incipit du conte : Beck suit le déroulement linéaire du texte en recopiant quelques mots (ou lettres) sélectionnés, et en paraphrasant/condensant ce qu'il n'élide pas. L'horizontalité de la prose se renverse ainsi en verticalité (c'est un des premiers sens qu'on peut donner au titre du chant : «réversibilité»). La première phrase du conte en prose : 
Il était une fois, en plein hiver, quand les flocons descendaient du ciel comme des plumes et du duvet, une reine qui était assise et cousait devant une fenêtre qui avait un encadrement en bois d'ébène, noir et profond ${ }^{13}$.

se redresse ainsi :

En hiver des flocons descendent

comme des plumes

d'oiseau discret.

Femme à la fenêtre noire

Quant à l'avant-dernière phrase du $\mathrm{I}^{\mathrm{er}}$ paragraphe :

Bientôt après, elle eut une petite fille qui était blanche comme la neige, vermeille comme le sang et noire de cheveux comme le bois d'ébène, et Blanche-Neige fut son nom à cause de cela,

elle se condense ainsi :

Elle a bientôt une enfant à trois couleurs.

Une couleur lui donne son nom.

Les dialogues supprimés donnent parfois (rarement) lieu à d'expéditifs résumés, dès lors combinés avec le prélèvement et la paraphrase, comme dans la séquence où la reine est rendue malade de jalousie par la révélation du miroir :

Blanche-Neige cependant grandissait peu à peu et devenait toujours plus belle; et quand elle eut sept ans, elle était belle comme le jour et bien plus belle que la reine elle même. Et quand la reine, un jour, questionna son miroir :

Miroir, gentil miroir, dis moi, dans le royaume Quelle est de toutes la plus belle?

Le miroir répondit :

Dame la reine, ici vous êtes la plus belle, mais Blanche-Neige l'est mille fois plus que vous.

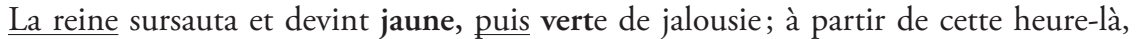
elle ne pouvait plus voir Blanche-Neige sans que le cœur lui châvirat dans la poitrine tant elle la haïssait. L’orgueil poussa dans son cœur, avec la jalousie, comme pousse la mauvaise herbe, ne lui laissant aucun repos ni de jour, ni de nuit.

La récriture ramasse en un vers le motif de la beauté adolescente: "L'enfant grandit. Elle est comme le jour» et résume prosaïquement, au vers suivant, le dialogue en vers de l'original, paraphrasé comme «inter-

13. En gras : les mots ou syllabes prélevés dans la traduction française; soulignés : les passages paraphrasés/condensés. 
rogatoire du miroir» (avec rime interne en miroir); le changement de couleur de la marâtre de l'original est intériorisé (récrit comme métamorphose morale), avec maintien par prélèvement desdites couleurs dans le lexique de l'original : «crée de nouvelles couleurs dans le cœur / [...] jaune et vert»; il en résulte un effet de contraste, créé par la sobriété du Chant, entre les couleurs de la jeune fille et celles attribuées à sa rivale, à laquelle Beck refuse en revanche tout au long d'accoler le terme "marâtre, " au profit de différentes périphrases ou substituts : "Mère Suivante (ou suivante)», "Mère successive», la «mère». Pour désigner son bouleversement, le verbe "tanguer» est préféré au verbe "chavirer", de même le "ventre» à la "poitrine», mais le mot "cœur» est conservé comme sujet (avec rime interne "couleurs/cœur»); du coup c'est un effet de nausée qui apparait ; la récriture fait enfin ressortir la dynamique d'une poussée intérieure d' "orgueil » (mot repris de l'original avec élision de «jalousie») : dynamique d'une entité morale qui répond à celle de la croissance en beauté de l'enfant. Beck souligne d'ailleurs le contraste par un jeu de couleur : "la mauvaise herbe» de l'original étant récrite "herbe sombre», laquelle fait antithèse à "comme le jour».

L'enfant grandit. Elle est comme le jour.

L'interrogatoire du miroir

crée de nouvelles couleurs dans le cour

de la mère suivante :

jaune et vert.

Cœur tangue dans le ventre.

Mère successive.

Orgueil pousse en elle,

comme herbe sombre.

Le vocabulaire de la beauté féminine a été supprimé du Chant alors qu'il circule en mainte occurrence depuis le début du conte : «en regardant la belle neige au-dehors", "c'était si beau ce rouge sur la neige», "une autre femme qui était très belle», "orgueilleuse de sa beauté», "la femme la plus belle» "Blanche-Neige [...] devenait toujours plus belle». On voit aussi que Beck focalise l'enjeu sur la transformation morale de la "mère suivante». C'est d'ailleurs le thème des mères qui se trouve au centre des 20 premiers vers dont seuls les vers 8-9 et I2 sont dévolus à l'enfant. 


\section{Du maternel}

À cet égard, il vaut la peine de reprendre les premiers vers du Chant pour entendre ce que la récriture a transformé du propos de l'original en prose.

En hiver des flocons descendent

comme des plumes

d'oiseau discret.

Femme à la fenêtre noire

donne trois gouttes de sang

à Neige.

C'est un coquelicot de soi,

aux pétales séparés.

Elle a bientôt une enfant à trois couleurs.

Une couleur lui donne son nom.
I

IO

Une quasi-paronomase fait rimer les vers I et 5 : «descendent/de sang", tandis que "de soi» dérive de "de sang». Le Chant fait donc entendre un parallélisme de la chute des flocons, de la tombée du sang, de la génération de soi (comme fleur produite par le «don» du sang à «Neige»). L'image $\mathrm{du}$ "coquelicot [...] / aux pétales séparés» est un ajout de la récriture (appelé par l'étymologie de "discret»). Sang de femme et neige tombante engendrent l'«enfant à trois couleurs». Beck paraît d'ailleurs oblitérer le masculin, réserve faite du vers $5 \mathrm{I}$ : «Elle est admirée d'un» — où le substantif brille par son absence. La nature neige, une femme perd son sang et c'est un don : voici une fleur de printemps en plein hiver. (Dans Perceval le Gallois, l'épisode des trois gouttes de sang sur la neige se déroule précisément au printemps : le Chant populaire réaffilie ainsi le conte à la légendaire association des "trois couleurs" par où le mythe discrètement cité réinscrit le conte en chant général ${ }^{14}$.) "Les Chant populaires ou contes lyriques sont des chants impersonnels non pas des chansons, fondés sur la légende de comportements anciens.» (Avertissement, p. 9.)

Mais le temps n'a pas qu'une direction : "Femme à la fenêtre noire» a donc une enfant virtuellement sans père (virtuellement car la version des Grimm occupe la mémoire, car aussi l' «oiseau discret» s'il était le roitelet à triple bandeaux — blanc et noir et couronne orange ${ }^{15}$ — ferait métaphore ou supplément au père oblitéré); on sait bien qu’à cet égard aujourd'hui, la

I4. Sur la diffusion de ce motif dans les recueils de contes avant les Grimm, voir P. Delarue et M.-L. Ténèze, Le Conte populaire français, Paris, Maisonneuve et Larose, 2002, p. 660.

I5. Je remercie Fabrice Thumerel de m’en avoir proposé l'idée.

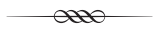


vie questionne vigoureusement le droit; quant à celle que le Chant refuse de nommer «marâtre», elle incarne toutes celles qui succèdent à — ... pour toutes sortes de raisons. À l'âge des «familles recomposées», qui trouverait malin d'employer ce vieux mot, si mal connoté? Et pourtant, c'est bien le mot des contes - et pas seulement de Blanche-Neige. Par la sélection orientée qu'il opère sur le conte des Grimm, Beck réembraye cette matière sur nos questionnements, focalisant l'intérêt sur l'histoire morale de la mère adoptive, ou de la mère "successive", de telle fille dite tellement "comme le jour» qu'elle provoque nausée, orgueil, sombre germination intérieure, "envie", désir de meurtre, passage à l'acte — et "cœur cuit». Il a aussi supprimé le châtiment de la danse en "escarpins de fer rouge» (rudesse sadique du merveilleux traditionnel : la «justice» de conte abonde en horreurs) : la torture est ici réinterprétée comme immanente au fait de la jalousie.

Reprenons la fin du conte :

Mais à ce grand mariage princier, la reine terrible et maudite marâtre de Blanche-

Neige fut invitée aussi; et quand elle se fut richement habillée et parée elle alla devant son miroir pour lui poser sa question :

Miroir, gentil miroir, dis-moi, dans le royaume

Qui est la femme la plus belle?

Et le miroir lui répondit :

\section{Dame la reine, ici vous êtes la plus belle, Mais la nouvelle reine est mille fois plus belle.}

Un juron échappa à l'horrible femme qui fut prise d'effroi, d'un tel effroi qu'elle ne savait plus que devenir. Pour commencer, son idée fut de ne pas aller du tout aux fêtes du mariage; mais elle ne put y tenir et il fallut qu'elle y allât, dévorée par la jalousie pour voir cette jeune reine.

Lorsqu'elle fit son entrée, elle reconnut ${ }^{*}$ immédiatement ${ }^{16}$ Blanche-Neige, et ${ }^{*}$ la peur qu'elle en eut la cloua sur place, ${ }^{*}$ sa terreur l'empêcha de bouger. Mais on lui avait préparé des souliers de fer qui étaient sur le feu, à rougir : on les lui apporta avec des tenailles et on les mit devant elle, l'obligeant à s'en chausser et à danser dans ces escarpins de fer rouge jusqu'à sa mort, qui suivit bientôt.

Ici la récriture est moins linéaire que dans les exemples précédents; ainsi, la séquence d'interrogatoire du miroir apparaît aussitôt après un résumé expéditif (traité en quasi-comptine) des trois tentatives meurtrières de «Mère suivante» :

I6. L’astérisque marque une transformation de l'intrigue ou de certains affects par le Chant. 
Mère suivante s'habille.

Elle vend du bel et bon.

Lacet coloré, peigne rond,

pomme à deux couleurs.

Blanche et rouge.

Blanche-Neige est presque morte,

ou morte officiellement. Décolorée. Miroir

dit la vérité froidement.

Et l'antichambre réelle.

Elle n'est pas dans la terre noire.

Elle est intacte dans le verre.

Alors que le conte souligne fortement la réalité de la mort de la jeune fille empoisonnée par la pomme ("elle tomba morte sur le plancher»), et secourue sans succès par les nains : "elle était vraiment morte», "morte elle était la chère petite, et morte elle resta", la récriture pose aussitôt ce que le lecteur/auditeur sait par cour: que cette mort n'est pas réelle, ou pas tout à fait : "presque morte/ou morte officiellement»; en outre, alors que le conte souligne que la mort de Blanche-Neige n'altère rien de sa beauté : "elle était encore aussi fraîche que si elle eût été vivante et elle avait encore toutes ses couleurs et ses belles joues rouges [...] elle avait l'air de dormir", Beck lui donne l'apparence du cadavre : "morte officiellement. Décolorée». Ironie du chiasme : le Chant scande tout le contraire du conte : mensonge de la fable officielle... Le fameux miroir omniscient ("Miroir/dit la vérité froidement») est dit "amagique», c'est-à-dire désenchantant (à l'inverse des écrans de notre médiasphère); savoir/dire vrai, rien de plus rare! Transposition quasi politique des Chants : «intempestivement maintenus dans un espace à la fois "classique” et prosaïque» (p. 9). Mais que reflète le miroir quand il est présenté au début du Chant?

Mère Suivante est peuplée.

Elle a un miroir qui dit si elle est singulière.

Miroir amagique.

Dans les entretiens déjà cités avec Gérard Tessier, Beck développe l'idée que l'individu singulier est une fiction, que chacun est un peuple : "Je contiens un peuple comme chacun. Il y a une mémoire involontaire de ce peuple en chacun. [...] La recherche de la langue, c'est l'effort pour décrire esthétiquement, ou inesthétiquement, l'élément commun en cours." (B. I., p. 83.) Qu'est-ce qui peuple Mère suivante? Classiquement, les passions de l'âme en clef d' "orgueil» (alias amour-propre dans la langue de l'augustinisme ou de Rousseau). Mais chez Beck, les personnages sont des mots dont la charge (l'emprise) peut être retravaillée, réorientée : "L'élision 
de l'article crée une prosopopée des abstractions et une mutation des noms communs en noms propres ou noms de personnages.» (Ibid., p. 58.) Ainsi :

Cour tangue dans le ventre.

Mère successive.

20

Orgueil pousse en elle,

comme herbe sombre.

[...]

envie a brûlé ses mouvements.

\section{Démoraliser/rédifier : de la prosopopée}

Prosopopée de Mère successive, de Cœur, d'Orgueil, d' «envie»; comme dans le Roman de la rose et dans la lyrique ancienne; comme chez Baudelaire : "Sois sage ô ma Douleur...». Douleur de Mère successive, nausée d'envie (au vieux sens) balancée en brefs mètres irréguliers. Elle naît à son peuple intérieur dans l'amagie du miroir de vérité. Et ce peuple affronte le peuple de l'Autre. Chez les Grimm, il n'est question que d'une rivalité de beauté entre proches-lointaines; dans la récriture il n'est question que de singularité : «Elle a un miroir qui dit si elle est singulière.» Or elle se révèle plurielle : peuplée au plus intime de soi : ce Chant médite une initiation à soi comme produit de la rencontre avec l'autre en soi, dehors/dedans. "Le dehors est peuplé comme un dedans. Il est comme les rues d'Atget : elles attendent de nouveaux locataires. En mémoire des anciens, insuffisants.» (B. I., p. 63.) Beck a pour cela une image : «Le moi est un gant, comme Intériorité. [...] La prose intérieure, c'est la prose du malheur des gens, mais rentrée, réversible. Intériorité est un fleuve d'oubli réversible.» (B. I., p. 225.) C'est cette réversibilité de soi à soi qui advient au miroir amagique de Mère suivante (qui est ici, selon la formule lacanienne, signifiant pour un autre signifiant : mot pour mot, figure de soi) : "Les mots, écrit Beck, sont des impersonnages dans le poème progressif.»(B. I., p. IO3 et IoI.)

Dans le Chant, il est dit quelque chose de la prosopopée : cette figure qui «met en scène les absents, les morts, les êtres surnaturels ou même les êtres inanimés, pour les faire agir, parler, répondre» (Fontanier, Les Figures $d u$ discours). C'est à l'endroit où la récriture élide/condense presque tout de la première tentative de meurtre par l'entremise du chasseur royal, ainsi que du stratagème de la substitution des poumons et du foie du marcassin à ceux de la jeune fille, pour le dîner de la "mauvaise femme» (comme dit la traduction). Voici ce que narre le Chant : 
Au loin dans la forêt, enfant comme le jour est laissée.

Pompe animale est humanisée.

Pompe de discours et désir.

Dedans remplacé.

Réaffecté.

Prosopopée de «Pompe» et de «Dedans»: métaphore technique du fameux "poumon" marcassin substitué (chez Grimm) à celui de Neige (déplacement prosaïque du topos du cour mangé). Mais "Pompe» se dit aussi d'une rhétorique, d'un style, d'un apparat : "Pompe de discours et de désir» : pompes et œuvres du Mal? Vanité des vanités? Mère suivante est repeuplée : «Dedans remplacé/réaffecté».

Chants sont des contes refaits.

Des rédifications.

Morale vient dans la suggestion.

C'est ce qu'on lit aux premiers vers de l'Ouverture, en tête du recueil. Réfection et réaffectation : les Chants populaires ont une visée didactique. "Rédification» qui s'accomplit au présent de l'indicatif. Systématiquement (trait général de l'écriture de Beck, en vers comme en prose). Cette actualisation temporelle débraye radicalement cette histoire du temps révolu où confine le système imparfait-passé-simple propre au récit littéraire. Quand Beck en parle, il noue cela à l'articulation du philosophique et du poétique : «Le poème lyrique est un poème au présent. Ce qui hante le texte philosophique est le présent. D'où un problème avec l'imparfait et le passé simple, les temps du récit de la marquise commune.» (B. I., p. 4I.) Mère suivante et Neige : leur réversibilité, c'est ici-maintenant, dans un chant impersonnel où "un présent générique détermine la capacité négative de contes qui ne sont pas des fables", précise Beck (Avertissement, p. 9).

"Et le cœur de la "mère" / est cuit»; cœur de celle entre guillemets, désormais en quasi-suspens d'être; réplique, à cet endroit du Chant, de la "presque morte» par son fait : Réversibilité. Ce que la récriture avait élidé de la cuisine royale anthropophage chez les Grimm : «il fallut que le cuisinier les mît au sel et les fit cuire», a consumé la pompe cordiale de l'Envie incarnée. Il y aura fallu tout le Chant : "envie a brûlé ses mouvements». La récriture est une solution herméneutique : la danse de mort en escarpins d'Enfer est lue comme allégorie : telle "Mère suivante» est consumée de passion mimétique. Ce mimétisme se tisse dans la trame du chant par le double jeu de forge rythmique et de "phonerie» (un des néologismes de Beck) : «envie a brûlé ses mouvements», est-il écrit de 
«mère»; «mouvements" fait loupe à tout ce qui se dit, depuis une douzaine de vers, de la vérité de la vie perdurante de la rivale, à l'envers de laquelle se consume sa propre vie de moins-belle-désormais, prise au "dur désir de durer» (Éluard), récrit comme "vie dure» par le Chant. Comment se lit un poème? "Littéralement et dans tous les sens", soit réversiblement selon Beck (ou Mallarmé) : "La mémoire spéciale du poème crée un horizon tombant. Les choses se disent dans le poème de bas en haut et de haut en bas dans la relecture. Le repassage du bas au haut et de la fin au commencement. La relecture est originaire et officielle par poème.» (B. I., p. 217.) Allons-y :

\section{Miroir}

froidement.

infiniment.

aime immédiatement;

brève suite de moments

commence l'élan.

Et le cœur de la «mère»"

enVIE a brûlé ses mouvements.

VIE durre.

Le miroir amagique ne ment pas : froidement, il dit la vérité (rythmiquement); une vérité cuisante au cœur de "mère" (et le rythme s'y casse en mesure). C'est de la vie de celle qui est «intacte», qui n'est pas enterrée, et qui aime, etc., que parle le miroir où "mère " accède en concentré à l'incendie qui consume "vie dure» (vie qui endure, vie qui dure, vie dure à -), par où l'on sent bien que l'on n'a pas ici affaire à un «cas", mais à un fragment d'universel : "Poème est le nom d'un récit général égal au raisonnement sensible dont chacun est capable. Chacun en est capable mais chacun est aussi l'oubli de ce poème dans la nuit quotidienne. [...] Poème est enseignement ou rappel de la condition humaine.» (B. I., p. 79.)

\section{Réversibilité}

Le titre du conte de Grimm est un pléonasme - ce que révèle non sans humour celui de la récriture; mais Beck y lit aussi une métonymie : "Elle a bientôt une enfant à trois couleurs. / Une couleur lui donne son nom.» Le Chant populaire inscrit la figuralité du Chant comme son propre. "Neige» paraphrase au v. 5 la tombée des flocons, tout en inscrivant la/ le destinataire du don du sang de "femme à la fenêtre noire». Comme avec le vocable «marâtre», Beck refuse, 4I vers durant, de livrer le fameux 
nom «Blanche-Neige», ne le laissant s'inscrire — et une seule fois — qu’à l'occasion de sa presque-mort. Au défaut parait d'abord son nom (issu) de couleur — d'ailleurs seulement suggérée au v. 9-; vient ensuite "enfant comme le jour", citation abrégée où se marque le manque du signifiant de la beauté; puis survient "Neige» au v. 27 : "Neige semble éliminée», par où revient la prosopopée du v. 5 mais en mode disparaissant; puis très vite, une paraphrase fulgurante d'une image où le conte figure la terreur : «Mais dans la forêt, / Neige retourne les feuilles. Nuit tombe.» Guerne proposait : "la malheureuse fillette était désespérément seule et tellement apeurée qu'elle regardait pour ainsi dire derrière chaque feuille sur les arbres ", et nous suivions la course panique de la jeune fille dans la forêt des contes; dans les vers de Beck, le pathos est annulé au profit d'une antithèse "Neige/Nuit». Bientôt paraissent «Nappe blanche et draps blancs" sur fond de «Nuit noire».

La suite du Chant met en parallèle les couleurs de la pomme empoisonnée et le nom-de-conte de l'héroïne : «Pomme à deux couleurs. Blanche et rouge. / Blanche-Neige est presque morte.» Mais ce nom-pléonasme contient par métonymie du noir d'ébène. Dans ses maléfices, Mère suivante manque de noir absolu (selon la récriture, même sa couleur interne n'est pas le noir mais un jaune-vert nauséeux); elle manque de ce qui doue Neige depuis l'encadrement de la «fenêtre noire» où telle femme l'a conçue. Quant à la "presque morte» qui n'est plus désignée que comme l'Autre du miroir par pronom en italique, elle échappe à la mort par la transparence qui la résume depuis son nom «Elle n'est pas dans la terre noire / Elle est intacte dans le verre»; c'est ainsi comme «Blanche» qu'elle est alors nommée (c'en est la seule occurrence), par l'amour «d'un»: "Blanche» qui assonne avec "officiellement», « infiniment», «immédiatement», "commence», "élan» — et ce qui s'ensuit pour "mère» recevant enfin la couleur qui lui manque, puisque «cœur cuit» c'est cœur en cendre. Les Grimm, pour leur part, préférèrent un contraste BlancheNeige/rouge ardent. Ici le dedans, là le dehors. Réversibilité.

\section{Une forme qui exprime le gâchis}

Dans un entretien récent, Philippe Beck considère que «les poèmes se périphrasent eux-mêmes ${ }^{17} »$. Une façon de prendre congé serait de demander

17. Entretien avec M. Rueff et Th. Samoyault à propos de Lyre dure, disponible sur <http:// www.m-e-l.fr/rencontres-publiques.php?id=I24> [consulté le 8 septembre 20IO]. 
comment ici le Chant commente le Chant; dans le choix du titre sans doute, et l'allure générale d'un poème où les noms sont au générique des personnages. Bien. Mais une remarque de Jacques Rancière à propos de l'écriture de Beck peut relancer le jeu :

C'est sur ce mode de la fable que Philippe Beck nous parle du «règne animal de l'esprit» $[\ldots]$ car «fable» justement veut dire cela : non pas simplement des histoires d'animaux mais une pratique du langage qui actualise une idée du langage [...], une manière de dire une chose pour en dire une autre ${ }^{18}$.

Il faudra donc rectifier le différend conte/fable postulé (à la suite de Beck) au début de cette étude : car si tous les dialogues ont été supprimés, une voix s'insinue en commentaire — passablement décalée : "Neige retourne les feuilles. Nuit tombe. / Elle trouve une maison / Miniature. Comme Alice? / Nappe blanche et draps blancs dedans. / Est-ce le Hollandais Volant? / Un navire à bascule?» Une voix commente, mais avec quelle idée derrière la tête? La «maison/miniature» est un prélèvement sur la traduction de Guerne, mais le rejet et la comparaison la réinscrivent chez Lewis Caroll : façon de relancer la question du merveilleux? de la recartographier dans notre géographie mentale? La légende du Hollandais Volant est un mythe réactualisé, outre les fastes wagneriens, par l'extraordinaire Pandora (195I) d'Albert Lewin; qu' est-ce que cette histoire de vaisseau fantôme vient faire dans cette forêt de récriture désertée par les sept nains? De quelle couleur la chevelure d'Ava Gardner? Et qui au juste est presque mort dans cette histoire? réversibilité? Et puis, qu'est-ce qui fait tanguer cœur au ventre chez Mère suivante? "Un navire à bascule» où chavirent les cours?

Vaisseau pirate de foire:

La prose du monde de maintenant est chaotique, répond Beck. Il faut méditer et reméditer les mots de Beckett. Je les recopie ici : "Comment admettre le gâchis, qui semble être le contraire même de la forme? [...] Trouver une forme qui exprime le gâchis, telle est maintenant la tâche de l'artiste." ( $M d A$, p. 45.)

Foirer mieux, c'est peut-être l'humour noir des crimes manqués de Mère suivante. Mais dans «Blanche» et dans "Neige» joue une réversibilité lexicale inconnue des frères Grimm : lire Blanche-Neige après, mettons Le Festin nu ou tel film éponyme de Juliet Berto : Vie flambe à Manque - à Mort ${ }^{19}$.

I8. J. Rancière, "Note sur la bouphonie transcendantale», Il particolare, ${ }^{\text {os }} 7$ et 8, 2002. Cité dans B. I., p. 24I.

19. Merci à Robert Briatte pour cette piste. 
Sur ce circuit, on rencontre nécessairement le peintre de la vie moderne, de sa beauté classique comme un conte de Grimm et bizarrement terrible comme un Chant populaire de Beck : et c'est à lui qu'on veut laisser le mot de la presque-fin, comme auteur d'une suite de strophes éprouvantes, dont notamment celle-ci :

Ange plein de beauté, connaissez-vous les rides,

Et la peur de vieillir, et ce hideux tourment

De lire la secrète horreur du dévouement

Dans des yeux où longtemps burent nos yeux avides?

Ange plein de beauté, connaissez-vous les rides?

On saura aisément en retrouver le titre. 